\title{
Lupus Sclerosing Peritonitis
}

\author{
Talib S.H*, Sainani Rahul**, Deshpande Shreyas*** \\ *Professor \& Head $\quad * *$ Chief Resident, $* * *$ Senior Resident \\ Department of Medicine, Mahatma Gandhi Missions Medical College \& Hospital, Aurangabad.431003
}

\begin{abstract}
Lupus manifesting as an ascites is extremely rare as aptly assessed by the figures available in world literature. We report a case which clinically and cytologically mimicked a malignant process. Further investigations and conservative approach taken in the case led to definitive diagnosis of florid peritoneal reaction associated with systemic lupus erythematosus. The clinical presentation is discussed in the context of other similar rare reports.
\end{abstract}

Key words : Fibrosis, sclerosing peritonitis, florid ascites, systemic lupus erythematosus.

\section{Case Report :}

A 59 years old male patient presented to us in January 2009 for massive central abdominal ascites with abdominal discomfort and exertional dyspnoea of 6 weeks duration. There was no previous history of medication use, radiation or asbestos exposure, drug abuse, abdominal surgery, trauma or infection. Three weeks prior to the present hospitalization he was investigated for subacute onset of gross ascites at private nursing home. His haemogram, KFTs, LFT's (Bilirubin 1.1 direct : 0.3, indirect : 0.8, SGOT: 42, SGPT : 38, AP: 761) reported as normal. CT chest and abdomen were showing bilateral effusion and ascites. Cardiac 2D echo done showed mild concentric LVH, no regional wall motion abnormality, no evidence of clot, vegetation or any pericardial pathology, the ejection fraction was $87 \%$.

Ascitic fluid microscopy revealed no cobweb, TLC was 1400/cumm with plenty of RBC and lymphocytes $90 \%$, protein contents were $3.8 \mathrm{gm} \%$ smears were negative for Acid fast bacilli and malignant cells. Polymerase Chain Reaction (PCR) for Mycobacterium tuberculosis was negative. Patient was hospitalized on 28/1/2009 for further investigations and management. The physical examination revealed gross ascites, without evidence of organomegaly or peripheral clinical stigmata of liver disease. He had no signs of arthritis or any skin lesion. There was ++ dependent oedema on legs, JVP was not raised, there was no lymphadenopathy, all vital parameters were well maintained. Other systemic examination was unremarkable.

Analysis of ascitic fluid revealed protein $4.3 \mathrm{gm} \%$ total count of fluid was 200/cumm with $80 \%$ lymphocytes, smear also showed degenerative mesothelial cells. Ascitic ADA was 7.9 U/L. Repeat PCR for mycobacterium tuberculosis of pleural and ascitic fluid were negative. ECG was normal. Enhanced and unenhanced serial axial sections of abdomen and pelvis were undertaken. CT revealed moderate thickening (9$10 \mathrm{~mm}$ ), nodularity and post contrast enhancement of omentum and peritoneum along with moderate ascites. The bowel loops were adherent to thickened peritoneum, no obvious bowel wall thickening was noted (Fig.1,2). No obvious lymphadenopathy noted within the abdomen and pelvis. An attempted CT biopsy of omentum and peritoneal cakes revealed many inflammatory cells predominantly polymorphs and few lymphocytes. Also seen were moderate size clumps of round oval nuclei. There was moderate to severe anisocytosis and anisonucleosis, possibility of malignancy could not be ruled out.

Considering possibility of Gastrointestinal malignancy OGD scopy done was normal. Tumour markers CEA $0.9 \mathrm{ng} / \mathrm{ml}$ (normal 0-7.4 ng/ml), CA 19-9 was 2.8U/ml (ref upto $37 \mathrm{U} / \mathrm{ml}$ ), AFP $1.48 \mathrm{ng}$ (ref upto $20 \mathrm{ng}$ ). SAAG score of ascitic fluid was $>1.1$. The colour Doppler of hepatic vein was normal, there was no evidence of thrombosis, ruling out the possibility of Budd Chiari syndrome. Subsequent investigations revealed that ANA was 1.1 (>1 positive) and Anti dsDNA was 72IU/1 (normal upto 42). The serologic abnormalities were considered diagnostic of Lupus peritonitis. Patient was treated with Tab.Azathioprine $100 \mathrm{mg}$ od, Inj. Methylprednisolone 125mg IV 8 hrly and Tab.Tamoxifen $20 \mathrm{mg}$ od. Patient was well until 7 days. One morning while in washroom he became tachypnic, cyanosed and collapsed. ECG taken at this moment showed sinus tachycardia change in frontal axis to complete RBBB. In a short period patient succumbed to death. Post mortem consent was not available.

\section{Discussion :}

Lupus peritonitis manifesting as florid ascites is an uncommon presentation. Review of literature revealed only 15 case reports of SLE associated chronic peritonitis.1,2 The ascites being predominant presenting feature is recorded only in 5 cases until 1996.3 Chronic lupus peritonitis presents a diagnostic problem in 
particular when huge ascites is predominant and is only the presenting feature of Systemic Lupus Erythematosus, as found in the present case and six other earlier reported cases.3,4 Pepels et al in 2006 reported a case of sclerosing peritonitis as an unusual cause of ascites in a patient with systemic lupus erythematosus. 2

The present case was particularly unusual in that patient had manifestatio ns of huge ascites besides having central distension of abdomen. The $\mathrm{CT}$ abdomen enhanced and unenhanced serial sections revealed moderate thickening of $10-11 \mathrm{~mm}$ caking and nodularity of omentum and peritoneum along with massive ascites. The bowel loops were adherent to thickened peritoneum and omentum, leading to central distensibility. Additionally patient had bilateral pleural effusion (right >left). Patient had no signs of intestinal obstruction. This finding along with the clinical history of rapidly developing ascitis in the absence of any obvious known etiologies, raised the consideration of carcinomatosis.

Finney et al in 1996 in his review on the subject has reported three cases of lupus peritonitis with adhesions and only one of these found to have dense, matting together loops of bowel besides his own case where histopathology was confirmative of sclerosing peritonitis. 3

Pepels and co-workers in 2006 claimed that their patient had recurrent ascitis with sclerosing lupus peritonitis as first ever case reported in literature.2

The cytology of ascitic fluid and FNAC from thickened mesentary was described as doubtful expression of concern for malignancy. Tumor markers like CEA, CA 19.9, PSA, AFP were all uncontributing in the present case. Anti $\mathrm{HCV}$, serum amylase, HBsAg tests were also negative. Similar suspicious features of malignancy were also reported in the case reported by Finney and co workers in 1996, however in the case clinical, cytological, serological and radiological features were more in favour of reactive process. The author proposed the term 'pseudosarcomatous sclerosing peritonitis', appropriate to emphasis the cellular nature of reaction and the potential for misdiagnosis of malignant process. 3

Sclerosing peritonitis resulting from other causes may also

have similar gross appearances with thickening of peritoneum, formation of plaque, adhesions, fibrosis and bowel adherence.5,6,7 Efforts are needed both clinically and pathologically/serologically to exclude various known possibilities of etiologic factors these include drugs like beta blockers, silica exposure, infections, granulomatous disorders, previous surgery with reaction to starch, past history of peritonitis secondary to leaks of bile/urine/intestinal perforations.8,9

The condition is also described in patients who have undergone peritoneal dialysis, in patients who received intraperitoneal chemotherapy, $\mathbf{1 0}$ it may be an expression of paraneoplastic phenomenon $\mathbf{1 1}$. None of the above etiologies and conditions applied to our case. In the present case work up with haematologic and serologic abnormalities including high titres of Anti dsDNA antibodies were noted and the diagnosis was established after exclusion of all other considered etiologies and close conditions.

The overall prognosis is poor often with progression of fibrosing and sclerosing process. The bowel loop is ultimately involved and incased in fibrous cocoon resulting in sclerosing encapsulating peritonitis. This occurs in $90 \%$ of the patients, the situation often leads to a significant morbidity due to bowel obstruction and sepsis with mortality rate upto $80 \% .2$

Most such patients were treated with Prednisolone $30-50 \mathrm{mg} /$ day, some cases supplemented with Azathiprime 100-125 mg/day. The outcome remain difficult to interpret because of efficacy depended on the stage of patients 12 Tamoxifen $40 \mathrm{mg} /$ day for a mean of 14 months resulted in prevention of encapsulated peritoneal sclerosis which significantly lowered the mortality (22\% vs 71\%).8,13 The drug shows reduction in thickness of peritoneum perhaps induced by angiogenesis inhibitions.14,15 Our case received Prednisolone, Azathioprine and Tamoxifen in the prescribed doses .Patient was doing well for nearly one week and found to have sudden acute onset tachypnea, cyanosis and hypotension while in washroom. He was noted to have clinical and electrographic evidence of pulmonary infarction and later succumbed to death.

Pepels and co workers in 2006 also reported similar case who had progressive onset of dyspnea. The CT angiography revealed in the case evidence of pulmonary embolism. The patient was duly put on acinocoumarol, the echo duplex of leg veins showed no venous thrombosis. 2 Lupus patients are at significantly increased risk for premature atherosclerosis and thrombosis which is a multifactorial process. The thrombosis risk assessment in lupus require lupus disease activity/severity, acquired and genetic risk factor and Antiphospolipid (APL) profile.16 Further a significant APL profile may not be clinically relevant in every SLE patient, and it is common for patients with SLE to develop thrombosis in absence of APL.17,18

It is apparent that this rare, serious and unusual case require exclusions of variety of possible etiologic factors when faced with such type of entity. The disease needs more explorations in its cellular nature of reactions, investigative modalities, therapeutic and in preventive options.

We humbly concede that lupus peritonitis presenting as florid ascitis is unheard in Indian literature. 


\section{References:}

[1]. Kaklamonis P, Vayopoulos G, Stamatelos G, Dadinas G,Tsokos GC. Chronic lupus peritonitis with ascites. Ann Rheum Dis. 1991; $50: 176-177$.

[2]. Pepels M.J.A.E. Peters F.P.J. Mebis J.J.I.R., Ceelen Th L. Hoofwijk A.G.M., Erdkamp F.C.G. Sclerosing peritonitis : an unusual cause of ascites in patients with systemic lupus erythematosus. Netherlands Journal of Medicine. 2006; 64(9): 346-349.

[3]. Finney AL, Spagndo DV, Crawford GP, Shilkin KB. Pseudosarcomatous sclerosing peritonitis. Case report of an unusual form of chronic lupus peritonitis. International Journal of Surgical Pathology. 1996; 4(2) : 121-128.

[4]. Wilkins K, Holffman G. Massive ascites in systemic lupus erythematosus. J Rheum. 1985; 12: 571-574.

[5]. Mier A, Weir M. Ascites in systemic lupus erythematosus. Ann Rheum Dis. 1985; 44 : 778-779.

[6]. Miller MH, Urowitz MB, Gladmann DD, Tozman ECS. Chronic adhesive lupus serositis as a complication of systemic lupus erythematosus. Arch Intern Med. 1984; 144 : 1863-64.

[7]. Schousboe J, Koch A, Chang R. Chronic lupus peritonitis with ascites : review of literature with a case report. Semin Arthritis Rheum. 1988; $18:$ 121-126.

[8]. Garosi G, Di Paolo N. Morphological aspects of peritoneal sclerosis. J Nephro. 2001; 14 (4) : 530-8.

[9]. Kelly JK, Hwary WS. Idiopathic refractile sclerosing mesenteritis and its differential diagnosis. AM J Surg Pathol. 1989; 13 : $513-$ 521.

[10]. Dehn TCB, Lucas MG Wood RFM. Idiopathic sclerosing peritonitis. Postgrad Med J. 1985; 61 : 841-841.

[11]. Vlasveld LT, Taal BG, Kroon BBR, Galle MPW, Rodenhuis S. Intestinal obstruction due to diffuse peritoneal fibrosis at few years after the successful treatment of malignant peritoneal mitoxantrone. Cancer Chemotheraphacol.1992; 29:405-408.

[12]. Michel C, Hufilagel G, Niang A et al. Sclerosing peritonitis Nephron. 2001; 22(4) : 141-8.

[13]. Del Peso G, Bajo M, Gil F et al. Clinical experience with Tamoxifen in peritoneal fibrosing syndromes. Adv Perit Dial. 2003; 19 : $32-5$.

[14]. Izumotani T, Ishimura E, Yamamoto T et al. Correlation between peritoneal mesothelial cell cytology and peritoneal histopathology with respect to prognosis on continuous ambulatory peritoneal dialysis. Nephron. 2001; 89(1): 43-9.

[15]. Allaria P, Giayrande A, Gandini E, Pisoni I. Continuous ambulatory peritoneal dialysis and sclerosing encapsulating peritonitis : Tamoxifen as a new therapeutic agent. J Nephrol. 1999; 12(6) : 395-7.

[16]. Erkan D. Ed. Lupus and Thrombosis. The Journal of Rheumatology 2006; 33:9.

[17]. Moc CC. Accelerated atherosclerosis, arterial thromboembolism and preventive strategies in systemic lupus erythematosus. Scand J Rheumatol 2006; $35: 85-95$.

[18]. Afeltra A, Vadacca M, Conti L et al. Thrombosis in systemic lupus erythematosus; congenital and acquired risk factors. Arthritis Rheum. 2005; 53 : 452-9.

\section{FIGURES :}
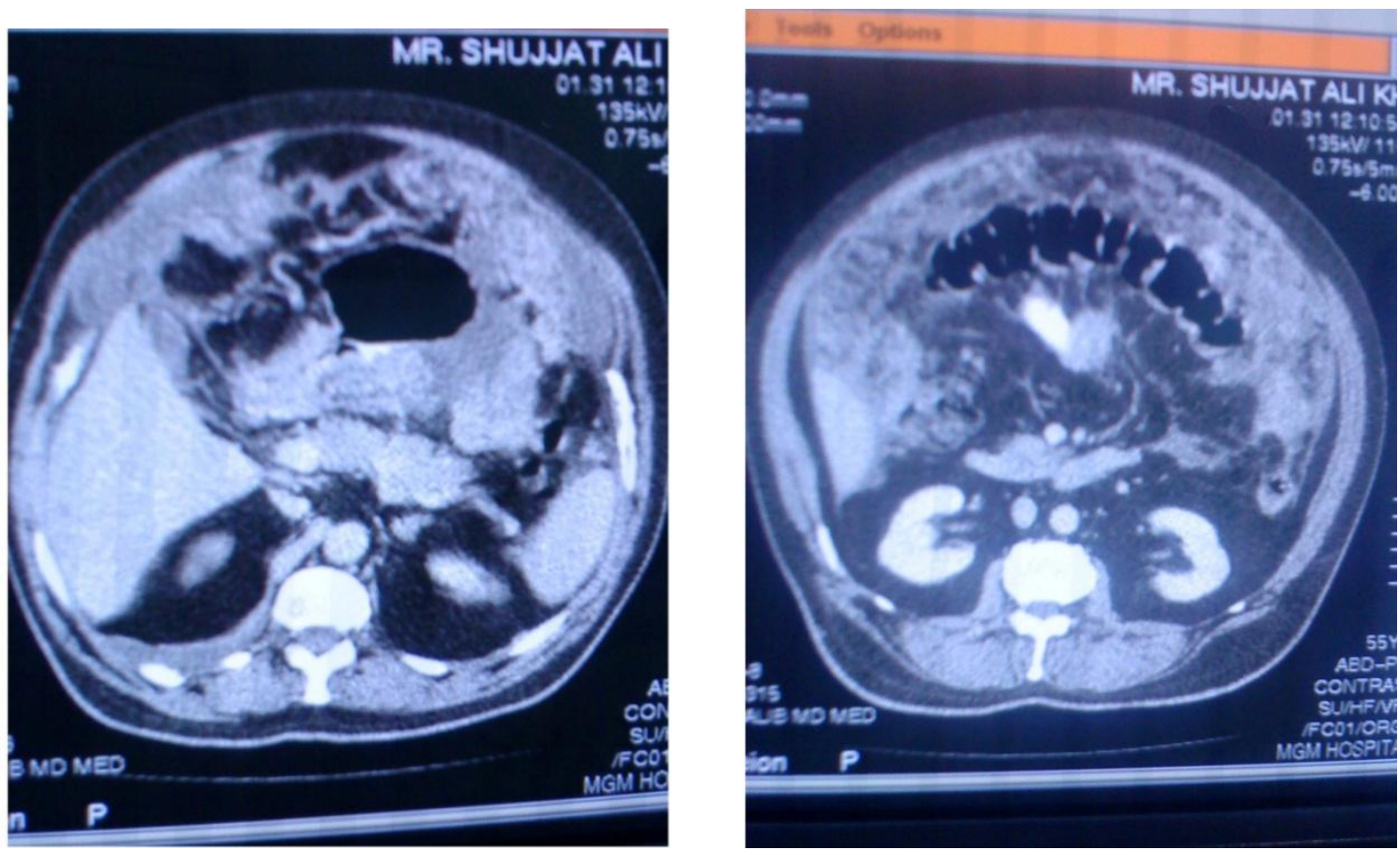

Figure-1 \& 2 : CT abdomen reveals thickening, nodularity of peritoneum with adhesions to bowel loops 